\title{
Strong Convergence of a Modified Ishikawa Iterative Sequence for Asymptotically Quasi-Pseudo-Contractive-Type Mappings
}

\author{
Liuhong Li and Yuanheng Wang \\ Department of Mathematics, Xingzhi College, Zhejiang Normal University, Zhejiang 321004, China \\ Correspondence should be addressed to Yuanheng Wang; wangyuanhengmath@163.com
}

Received 20 January 2014; Accepted 19 February 2014; Published 26 March 2014

Academic Editor: Li Wei

Copyright (c) $2014 \mathrm{~L}$. Li and Y. Wang. This is an open access article distributed under the Creative Commons Attribution License, which permits unrestricted use, distribution, and reproduction in any medium, provided the original work is properly cited.

\begin{abstract}
The purpose of this paper is to investigate the strong convergence problem of a modified mixed Ishikawa iterative sequence with errors for approximating the fixed points of an asymptotically nonexpansive mapping in the intermediate sense and an asymptotically quasi-pseudo-contractive-type mapping in an arbitrary real Banach space. The results here improve and extend the corresponding results reported by some other authors recently.
\end{abstract}

\section{Introduction and Preliminaries}

It is well known that fixed point theory has emerged as an important tool in studying a wide class of nonlinear elliptic systems and nonlinear parabolic systems, obstacle, unilateral, and equilibrium problems, optimization problems, theoretical mechanics, and control theory, which arise in several branches of pure and applied nonlinear sciences in a unified and general framework. This alternative formulation has been used to study the existence of a fixed point as well as develop several numerical methods. Using this idea, one can suggest some iterative methods for fixed points and study the convergence of their iterative sequences.

Throughout this paper, we assume that $E$ is a real Banach space, $E^{*}$ is its dual space, $\langle\cdot, \cdot\rangle$ is the dual pair between $E$ and $E^{*}$, and $D(T), F(T)$ denote the domain of a mapping $T$ : $D(T) \rightarrow E$ and the set of all fixed points of $T$, respectively.

Let $J: E \rightarrow E^{*}$ be the normalized duality mapping defined by

$$
\begin{array}{r}
J(x)=\left\{f \in E^{*}:\langle x, f\rangle=\|x\|\|f\|,\|f\|\right. \\
=\|x\|\}, \\
\forall x \in E .
\end{array}
$$

Definition 1. Let $G$ be a nonempty subset of a real Banach space $E$ and $T: G \rightarrow G$ a mapping.
(1) The mapping $T$ is said to be asymptotically nonexpansive, if there exists a number sequence $\left\{k_{n}\right\} \subset[1, \infty)$ with $\lim _{n \rightarrow \infty} k_{n}=1$, such that

$$
\left\|T^{n} x-T^{n} y\right\| \leq k_{n}\|x-y\|, \quad \forall x, y \in G, n \geq 1 .
$$

(2) The mapping $T$ is said to be asymptotically pseudocontractive, if there exists a number sequence $\left\{k_{n}\right\} \subset$ $[1, \infty)$ with $\lim _{n \rightarrow \infty} k_{n}=1$ and $j(x-y) \in J(x-y)$, such that

$$
\left\langle T^{n} x-T^{n} y, j(x-y)\right\rangle \leq k_{n}\|x-y\|^{2}, \quad \forall x, y \in G, n \geq 1 .
$$

(3) The mapping $T$ is said to be uniformly $L$-Lipschitzian, if there exists a constant $L \geq 1$, such that

$$
\left\|T^{n} x-T^{n} y\right\| \leq L\|x-y\|, \quad \forall x, y \in G, n \geq 1 .
$$

Remark 2. It is easy to see that if $T: G \rightarrow G$ is an asymptotically nonexpansive mapping, then $T$ is uniformly $L$-Lipschitzian $\left(L=\sup _{n \geq 1}\left\{k_{n}\right\}\right)$; and if $T$ is an asymptotically nonexpansive mapping, then $T$ is an asymptotically pseudocontractive mapping. But the converse is not true in general. This can be seen from the example in [1].

In 1972, Goebel and Kirk [2] introduced the concept of asymptotically nonexpansive mappings which was closely 
related to the theory of fixed points of mappings in Banach spaces. Nine years later, the concept of asymptotically pseudocontractive mapping was introduced by Schu [3] in 1991. The iterative approximation problems for asymptotically nonexpansive mappings and asymptotically pseudocontractive mappings were studied extensively by many authors; see [1, 4-11]. The concept of asymptotically nonexpansive mapping in the intermediate sense was introduced and studied by Bruck et al [12]. When $D(T)$ is bounded, the class of asymptotically nonexpansive self-maps is the special case of the class of asymptotically nonexpansive self-maps in the intermediate sense.

Definition 3 (see [12]). Let $T: G \rightarrow G$ be a mapping, if for each $x, y \in G$, there holds the inequality

$$
\limsup _{n \rightarrow \infty}\left\{\sup _{x, y \in G}\left(\left\|T^{n} x-T^{n} y\right\|-\|x-y\|\right)\right\} \leq 0,
$$

then $T$ is called an asymptotically nonexpansive mapping in the intermediate sense.

The concept of asymptotically pseudocontractive-type mapping was first introduced by Zeng [13] in 2004. On this basis, the asymptotically quasi-pseudo-contractive-type mapping can be given as follows.

Definition 4. Let $T: G \rightarrow G$ be a mapping.

(1) The mapping $T$ is said to be of asymptotically pseudocontractive type, if there exists a number sequence $\left\{k_{n}\right\} \subset[1, \infty)$ with $\lim _{n \rightarrow \infty} k_{n}=1$, such that

$$
\begin{aligned}
& \lim \sup _{n \rightarrow \infty}\left\{\operatorname { s u p } _ { x \in G } \operatorname { i n f } _ { j ( x - y ) \in J ( x - y ) } \left[\left\langle T^{n} x-T^{n} y, j(x-y)\right\rangle\right.\right. \\
&\left.\left.-k_{n}\|x-y\|^{2}\right]\right\} \leq 0, \quad \forall y \in G .
\end{aligned}
$$

(2) The mapping $T$ is said to be of asymptotically quasipseudo-contractive type, if there exists a number sequence $\left\{k_{n}\right\} \subset[1, \infty)$ with $\lim _{n \rightarrow \infty} k_{n}=1$, such that

$$
\begin{array}{r}
\limsup _{n \rightarrow \infty}\left\{\operatorname { s u p } _ { x \in G } \operatorname { i n f } _ { j ( x - p ) \in J ( x - p ) } \left[\left\langle T^{n} x-p, j(x-p)\right\rangle\right.\right. \\
\left.\left.-k_{n}\|x-p\|^{2}\right]\right\} \leq 0, \\
\forall p \in F(T) \neq \emptyset .
\end{array}
$$

Remark 5. From the Definitions 1 and 4, it is easily known that the class of asymptotically quasi-pseudo-contractivetype mappings contains that of the asymptotically nonexpansive mappings with fixed points, the asymptotically pseudocontractive mappings with fixed points, and asymptotically pseudocontractive-type mappings with fixed points.
Definition 6 (see $[4,5]$ ). (1) Let $T: G \rightarrow G$ be a mapping, $G$ a nonempty convex subset of $E, x_{0} \in G$ a given point, and $\left\{\alpha_{n}\right\}$, $\left\{\beta_{n}\right\},\left\{\delta_{n}\right\}$, and $\left\{\gamma_{n}\right\}$ four number sequences in $[0,1]$. Then the sequence $\left\{x_{n}\right\}$ defined by

$$
\begin{aligned}
x_{n+1} & =\left(1-\alpha_{n}-\gamma_{n}\right) x_{n}+\alpha_{n} T^{n} y_{n}+\gamma_{n} u_{n}, \\
y_{n} & =\left(1-\beta_{n}-\delta_{n}\right) x_{n}+\beta_{n} T^{n} x_{n}+\delta_{n} v_{n}, \quad \forall n \geq 0,
\end{aligned}
$$

is called the modified Ishikawa iterative sequence with errors of $T$, where $\left\{u_{n}\right\}$ and $\left\{v_{n}\right\}$ are two bounded sequences in $G$.

(2) If $\beta_{n}=0$ and $\delta_{n}=0, n=0,1,2, \ldots$ in (8), then $y_{n}=$ $x_{n}$, the sequence $\left\{x_{n}\right\}$ defined by

$$
x_{n+1}=\left(1-\alpha_{n}-\gamma_{n}\right) x_{n}+\alpha_{n} T^{n} y_{n}+\gamma_{n} u_{n}, \quad \forall n \geq 0,
$$

is called the modified Mann iterative sequence with errors of $T$.

The modified Ishikawa and Mann iterative sequences with errors were studied by Zeng. He [4] proved the strong convergence of the modified Ishikawa iterative sequence with errors for the uniformly L-Lipschitzian asymptotically pseudocontractive mapping in an arbitrary real Banach space with the bounded range of $T$. Zeng [4] investigated the strong convergence of the modified Ishikawa iterative sequence with errors for the non-Lipschitzian asymptotically pseudocontractive mapping in an arbitrary real Banach space and gave the necessary and sufficient condition that $\left\{x_{n}\right\}$ is bounded and $\left\|T^{n} x_{n}-x_{n}\right\| \rightarrow 0$.

In this paper, motivated by the above results, we introduce a strong convergence theorem of the modified Ishikawa iterative sequence with errors for approximating fixed points of asymptotically nonexpansive mapping in the intermediate sense and asymptotically quasi- pseudo-contractive-type mapping in an arbitrary real Banach space. The results here generalize and improve the recent results announced by many other authors to a certain extent, such as $[1,4,5,12,13]$.

In order to prove our main results, we need the following lemmas.

Lemma 7 (see [14]). Let E be a real Banach space and $J$ the normalized duality mapping. Then

$$
\|x+y\|^{2} \leq\|x\|^{2}+2\langle y, j(x+y)\rangle
$$

holds for all $x, y \in E$ and $j(x+y) \in J(x+y)$.

Lemma 8 (see [15]). Let $\varphi:[0, \infty) \rightarrow[0, \infty)$ be a strictly increasing function with $\varphi(0)=0$, and let $\left\{\theta_{n}\right\},\left\{\sigma_{n}\right\}$, and $\left\{\lambda_{n}\right\}$ be nonnegative real sequences, such that $\sigma_{n}=o\left(\lambda_{n}\right), \sum_{n=0}^{\infty} \lambda_{n}=$ $\infty$, and $\lim _{n \rightarrow \infty} \lambda_{n}=0$. Suppose that

$$
\theta_{n+1}^{2} \leq \theta_{n}^{2}-\lambda_{n} \varphi\left(\theta_{n+1}\right)+\sigma_{n}, \quad n \geq 0 ;
$$

then $\lim _{n \rightarrow \infty} \theta_{n}=0$.

\section{Main Results}

Theorem 9. Let $G$ be a nonempty convex subset of a real Banach space $E, T: G \rightarrow G$ be an asymptotically nonexpansive mapping in the intermediate sense and an asymptotically 
quasi-pseudo-contractive-type mapping with sequence $\left\{k_{n}\right\} \subset$ $[1, \infty), \lim _{n \rightarrow \infty} k_{n}=1$. Assume that $\left\{\alpha_{n}\right\},\left\{\beta_{n}\right\},\left\{\gamma_{n}\right\}$, and $\left\{\delta_{n}\right\}$ are four number sequences in $[0,1]$ satisfying the following conditions:

(i) $\alpha_{n}+\gamma_{n} \leq 1, \beta_{n}+\delta_{n} \leq 1$;

(ii) $\alpha_{n} \rightarrow 0, \beta_{n} \rightarrow 0, \delta_{n} \rightarrow 0$, when $\left.n \rightarrow \infty\right)$;

(iii) $\gamma_{n}=o\left(\alpha_{n}\right), \sum_{n=0}^{\infty} \alpha_{n}=+\infty$.

Let $x_{0} \in G$ be a given point and the sequence $\left\{x_{n}\right\}$ the modified Ishikawa iterative sequence with errors defied by (8). Then $\left\{x_{n}\right\}$ converges strongly to a fixed point $p \in F(T)$ if only if there exists a strictly increasing function $\varphi:[0, \infty) \rightarrow[0, \infty)$ with $\varphi(0)=0$ such that

$$
\begin{aligned}
\limsup _{n \rightarrow \infty} \inf _{j\left(x_{n+1}-p\right) \in J\left(x_{n+1}-p\right)}\left[\left\langle T^{n} x_{n+1}-p, j\left(x_{n+1}-p\right)\right\rangle\right. \\
\left.\quad-k_{n}\left\|x_{n+1}-p\right\|^{2}+\varphi\left(\left\|x_{n+1}-p\right\|\right)\right]
\end{aligned}
$$

$\leq 0$.

Proof (sufficiency). Since $T: G \rightarrow G$ is an asymptotically quasi-pseudo-contractive-type mapping, we know $F(T) \neq \emptyset$ and we can choose a point $p \in F(T)$.

Assume there exists a strictly increasing function $\varphi:[0, \infty) \rightarrow[0, \infty)$ with $\varphi(0)=0$ such that the inequality (12) is satisfied. We can let $\overline{\varepsilon_{n}}=\inf _{j\left(x_{n+1}-p\right) \in J\left(x_{n+1}-p\right)}\left[\left\langle T^{n} x_{n+1}-\right.\right.$ $\left.\left.p, j\left(x_{n+1}-p\right)\right\rangle-k_{n}\left\|x_{n+1}-p\right\|^{2}+\varphi\left(\left\|x_{n+1}-p\right\|\right)\right]$, $\varepsilon_{n}=\max \left\{\overline{\varepsilon_{n}}, 0\right\}+(1 / n)$, for all $n \geq 1$.

By the definition of infimum, there exists $j\left(x_{n+1}-p\right) \epsilon$ $J\left(x_{n+1}-p\right)$ such that

$$
\begin{gathered}
\left\langle T^{n} x_{n+1}-p, j\left(x_{n+1}-p\right)\right\rangle-k_{n}\left\|x_{n+1}-p\right\|^{2} \\
+\varphi\left(\left\|x_{n+1}-p\right\|\right)<\overline{\varepsilon_{n}}+\frac{1}{n} \leq \varepsilon_{n} .
\end{gathered}
$$

By using (12), we have $\limsup _{n \rightarrow \infty} \overline{\varepsilon_{n}} \leq 0$. Hence $\lim _{n \rightarrow \infty} \varepsilon_{n}=0$.

Since $\left\{u_{n}\right\}$ and $\left\{v_{n}\right\}$ are two bounded sequences, we can let

$$
M=\sup \left\{\left\|u_{n}-p\right\|,\left\|v_{n}-p\right\|\right\}<\infty .
$$

From (8) and (14), we have

$$
\begin{aligned}
\left\|x_{n+1}-p\right\|= & \|\left(1-\alpha_{n}-\gamma_{n}\right)\left(x_{n}-p\right) \\
& +\alpha_{n}\left(T^{n} y_{n}-p\right)+\gamma_{n}\left(u_{n}-p\right) \| \\
\leq & \left\|x_{n}-p\right\|+\alpha_{n}\left\|T^{n} y_{n}-p\right\|+\gamma_{n}\left\|u_{n}-p\right\|
\end{aligned}
$$

$$
\begin{aligned}
& \leq \quad\left\|x_{n}-p\right\|+\alpha_{n}\left(\left\|T^{n} y_{n}-p\right\|-\left\|y_{n}-p\right\|\right) \\
& \quad+\alpha_{n}\left\|y_{n}-p\right\|+\gamma_{n} M \\
& \leq\left\|x_{n}-p\right\|+\sup _{x, y \in G}\left(\left\|T^{n} y-T^{n} x\right\|-\|y-x\|\right) \\
& \quad+\alpha_{n} \|\left(1-\beta_{n}-\delta_{n}\right)\left(x_{n}-p\right) \\
& \quad+\beta_{n}\left(T^{n} x_{n}-p\right)+\delta_{n}\left(v_{n}-p\right) \|+\gamma_{n} M \\
& \leq \quad\left\|x_{n}-p\right\|+\sup _{x, y \in G}\left(\left\|T^{n} y-T^{n} x\right\|-\|y-x\|\right) \\
& \quad+\alpha_{n}\left\|x_{n}-p\right\|+\alpha_{n} \beta_{n}\left(\left\|T^{n} x_{n}-p\right\|-\left\|x_{n}-p\right\|\right) \\
& \quad+\alpha_{n} \beta_{n}\left\|x_{n}-p\right\|+\left(\alpha_{n} \delta_{n}+\gamma_{n}\right) M \\
& \leq 3\left\|x_{n}-p\right\|+2 \sup _{x, y \in G}\left(\left\|T^{n} y-T^{n} x\right\|-\|y-x\|\right) \\
& \quad+\left(\alpha_{n} \delta_{n}+\gamma_{n}\right) M .
\end{aligned}
$$

Since $T$ is an asymptotically nonexpansive in the intermediate sense, and $\alpha_{n} \rightarrow 0, \delta_{n} \rightarrow 0, \gamma_{n} \rightarrow 0,(n \rightarrow \infty)$, there exists $n_{1}$, such that for all $n \geq n_{1}$,

$$
\begin{gathered}
\sup _{x, y \in G}\left(\left\|T^{n} y-T^{n} x\right\|-\|y-x\|\right) \leq \frac{1}{3}, \quad \forall x, y \in G, \\
\left(\alpha_{n} \delta_{n}+\gamma_{n}\right) M \leq \frac{1}{3} .
\end{gathered}
$$

From (15), (16), we have

$$
\left\|x_{n+1}-p\right\| \leq 3\left\|x_{n}-p\right\|+1 .
$$

By virtue of (13), we have

$$
\begin{aligned}
\limsup _{n \rightarrow \infty} & \left\langle T^{n} x_{n+1}-p, j\left(x_{n+1}-p\right)\right\rangle \\
& -k_{n}\left\|x_{n+1}-p\right\|^{2}+\varphi\left(\left\|x_{n+1}-p\right\|\right) \leq 0 .
\end{aligned}
$$

Thus there exists $n_{2} \geq n_{1}$, such that for all $n \geq n_{2}$,

$$
\begin{aligned}
& \left\langle T^{n} x_{n+1}-p, j\left(x_{n+1}-p\right)\right\rangle \\
& \quad-k_{n}\left\|x_{n+1}-p\right\|^{2}+\varphi\left(\left\|x_{n+1}-p\right\|\right) \leq 1 .
\end{aligned}
$$

Hence

$$
\begin{aligned}
\varphi\left(\left\|x_{n_{2}}-p\right\|\right) \leq & k_{n_{2}-1}\left\|x_{n_{2}}-p\right\|^{2} \\
& -\left\langle T^{n_{2}-1} x_{n_{2}}-p, j\left(x_{n_{2}}-p\right)\right\rangle+1 \\
= & k_{n_{2}-1}\left\|x_{n_{2}}-p\right\|^{2} \\
& -\left\langle T^{n_{2}-1} x_{n_{2}}-x_{n_{2}}+x_{n_{2}}-p, j\left(x_{n_{2}}-p\right)\right\rangle+1 \\
\leq & \left(k_{n_{2}-1}-1\right)\left\|x_{n_{2}}-p\right\|^{2} \\
& -\left\|T^{n_{2}-1} x_{n_{2}}-x_{n_{2}}\right\|\left\|x_{n_{2}}-p\right\|+1 .
\end{aligned}
$$


Since $\varphi$ is a strictly increasing function, we obtain

$$
\left\|x_{n_{2}}-p\right\| \leq \varphi^{-1}\left(a_{0}\right)
$$

where $a_{0}=\left(k_{n_{2}-1}-1\right)\left\|x_{n_{2}}-p\right\|^{2}-\left\|T^{n_{2}-1} x_{n_{2}}-x_{n_{2}}\right\|\left\|x_{n_{2}}-p\right\|+$ $1>0$.

Now we claim that for all $n \geq n_{2}$,

$$
\left\|x_{n}-p\right\| \leq 2 \varphi^{-1}\left(a_{0}\right) \text {. }
$$

Indeed, when $n=n_{2}$, it is easy to know that the result has been established by (21). Assume if for $n \geq n_{2},\left\|x_{n}-p\right\| \leq 2 \varphi^{-1}\left(a_{0}\right)$ holds, we want to prove $\left\|x_{n+1}-p\right\| \leq 2 \varphi^{-1}\left(a_{0}\right)$. Reduction to absurdity, assume that $\left\|x_{n+1}-p\right\|>2 \varphi^{-1}\left(a_{0}\right)$. Then, since $\varphi$ is a strictly increasing function, we have

$$
\varphi\left(\left\|x_{n+1}-p\right\|\right)>\varphi\left(b_{0}\right)>0,
$$

where $b_{0}=2 \varphi^{-1}\left(a_{0}\right)$. Using (17), we obtain for all $n \geq n_{2}$,

$$
\left\|x_{n+1}-p\right\| \leq 3\left\|x_{n}-p\right\|+1 \leq 6 \varphi^{-1}\left(a_{0}\right)+1 \text {. }
$$

From Lemma 7 and (8), we have

$$
\begin{aligned}
\left\|x_{n+1}-p\right\|^{2}= & \|\left(1-\alpha_{n}-\gamma_{n}\right)\left(x_{n}-p\right)+\alpha_{n}\left(T^{n} y_{n}-p\right) \\
& +\gamma_{n}\left(u_{n}-p\right) \|^{2} \\
\leq & \left(1-\alpha_{n}-\gamma_{n}\right)^{2}\left\|x_{n}-p\right\|^{2} \\
& +2 \alpha_{n}\left\langle T^{n} y_{n}-p, j\left(x_{n+1}-p\right)\right\rangle \\
& +2 \gamma_{n}\left\langle u_{n}-p, j\left(x_{n+1}-p\right)\right\rangle \\
\leq & \left(1-\alpha_{n}-\gamma_{n}\right)^{2}\left\|x_{n}-p\right\|^{2} \\
& +2 \alpha_{n}\left\langle T^{n} y_{n}-T^{n} x_{n+1}, j\left(x_{n+1}-p\right)\right\rangle \\
& +2 \alpha_{n}\left\langle T^{n} x_{n+1}-p, j\left(x_{n+1}-p\right)\right\rangle \\
& +2 \gamma_{n}\left\langle u_{n}-p, j\left(x_{n+1}-p\right)\right\rangle .
\end{aligned}
$$

Now we consider the second term on the right side of (25),

$$
\begin{aligned}
& 2 \alpha_{n}\left\langle T^{n} y_{n}-T^{n} x_{n+1}, j\left(x_{n+1}-p\right)\right\rangle \\
& \quad \leq 2 \alpha_{n}\left\|T^{n} y_{n}-T^{n} x_{n+1}\right\|\left\|x_{n+1}-p\right\| \\
& \quad \leq 2 \alpha_{n}\left\|T^{n} y_{n}-T^{n} x_{n+1}\right\|\left(6 \varphi^{-1}\left(a_{0}\right)+1\right),
\end{aligned}
$$

$$
\begin{aligned}
\left\|T^{n} y_{n}-T^{n} x_{n+1}\right\|= & \left\|T^{n} y_{n}-T^{n} x_{n+1}\right\| \\
& -\left\|y_{n}-x_{n+1}\right\|+\left\|y_{n}-x_{n+1}\right\| \\
\leq & \sup _{x, y \in G}\left(\left\|T^{n} y-T^{n} x\right\|-\|y-x\|\right) \\
& +\left\|y_{n}-x_{n+1}\right\| .
\end{aligned}
$$

Using (8) and $\left\|x_{n}-p\right\| \leq 2 \varphi^{-1}\left(a_{0}\right)$ for all $n \geq n_{2}$, we have

$$
\begin{aligned}
\left\|y_{n}-x_{n+1}\right\|= & \| \beta_{n} T^{n} x_{n}-\beta_{n} x_{n}+\delta_{n} v_{n}-\delta_{n} x_{n} \\
& -\alpha_{n} T^{n} y_{n}-\gamma_{n} u_{n}+\alpha_{n} x_{n}+\gamma_{n} x_{n} \| \\
\leq & \alpha_{n}\left\|T^{n} y_{n}-x_{n}\right\|+\gamma_{n}\left\|u_{n}-x_{n}\right\| \\
& +\beta_{n}\left\|T^{n} x_{n}-x_{n}\right\|+\delta_{n}\left\|v_{n}-x_{n}\right\| \\
\leq & \alpha_{n}\left(\left\|T^{n} y_{n}-p\right\|+\left\|x_{n}-p\right\|\right) \\
& +\gamma_{n}\left(\left\|u_{n}-p\right\|+\left\|x_{n}-p\right\|\right) \\
& +\beta_{n}\left(\left\|T^{n} x_{n}-p\right\|+\left\|x_{n}-p\right\|\right) \\
& +\delta_{n}\left(\left\|v_{n}-p\right\|+\left\|x_{n}-p\right\|\right) \\
\leq & \sup _{x}\left(\left\|T^{n} y-T^{n} x\right\|-\|y-x\|\right) \\
& +\alpha_{n}\left[\left(1-\beta_{n}-\delta_{n}\right) x_{n}+\beta_{n} T^{n} x_{n}+\delta_{n} v_{n}-p\right] \\
& +\alpha_{n}\left\|x_{n}-p\right\| \\
& +\sup _{x, y \in G}\left(\left\|T^{n} x-T^{n} y\right\|-\|x-y\|\right) \\
& +2 \beta_{n}\left\|x_{n}-p\right\|+\gamma_{n} M+\gamma_{n}\left\|x_{n}-p\right\| \\
& +\delta_{n} M+\delta_{n}\left\|x_{n}-p\right\| \\
\leq & 3 \sup _{x, y \in G}\left(\left\|T^{n} x-T^{y}\right\|-\|x-y\|\right) \\
& +2\left(2 \alpha_{n}+\alpha_{n} \beta_{n}+2 \beta_{n}+\gamma_{n}+\delta_{n}\right) \varphi^{-1}\left(a_{0}\right) \\
& \left(\alpha_{n} \delta_{n}+\gamma_{n}+\delta_{n}\right) M . \\
& \\
&
\end{aligned}
$$

Since $T$ is an asymptotically nonexpansive mapping in the intermediate sense, and $\alpha_{n} \rightarrow 0, \beta_{n} \rightarrow 0, \delta_{n} \rightarrow 0, \gamma_{n} \rightarrow 0$, $(n \rightarrow \infty)$, we obtain

$$
\left\|y_{n}-x_{n+1}\right\| \longrightarrow 0
$$

Substituting (29) in (27), we have $\left\|T^{n} y_{n}-T^{n} x_{n+1}\right\| \rightarrow 0$. By virtue of (26), we know for all $n \geq n_{2}$,

$$
2 \alpha_{n}\left\langle T^{n} y_{n}-T^{n} x_{n+1}, j\left(x_{n+1}-p\right)\right\rangle \leq \alpha_{n} C_{n},
$$

where $C_{n}=2\left\|T^{n} y_{n}-T^{n} x_{n+1}\right\|\left(6 \varphi^{-1}\left(a_{0}\right)+1\right) \rightarrow 0(n \rightarrow \infty)$.

Next we make an estimation for the third term on the right side of (25). Using (13), we have

$$
\begin{aligned}
& 2 \alpha_{n}\left\langle T^{n} x_{n+1}-p, j\left(x_{n+1}-p\right)\right\rangle \\
& \quad \leq 2 \alpha_{n} \varepsilon_{n}+2 \alpha_{n} k_{n}\left\|x_{n+1}-p\right\|-2 \alpha_{n} \varphi\left(\left\|x_{n+1}-p\right\|\right) .
\end{aligned}
$$

Finally we estimate the last term on the right side of (25). Using (24) and $\gamma_{n}=o\left(\alpha_{n}\right)$, we assume that $\gamma_{n}=d_{n} \alpha_{n}$, $d_{n} \rightarrow 0,(n \rightarrow \infty)$, and we have

$$
\begin{aligned}
2 \gamma_{n}\left\langle u_{n}-p, j\left(x_{n+1}-p\right)\right\rangle & \leq 2 \gamma_{n}\left\|u_{n}-p\right\|\left\|x_{n+1}-p\right\| \\
& \leq 2 d_{n} \alpha_{n} M\left(6 \varphi^{-1}\left(a_{0}\right)+1\right)=h_{n} \alpha_{n},
\end{aligned}
$$

where $h_{n}=2 d_{n} M\left(6 \varphi^{-1}\left(a_{0}\right)+1\right) \rightarrow 0,(n \rightarrow \infty)$. 
Substituting (30), (31), and (32) in (25), we obtain

$$
\begin{aligned}
\left\|x_{n+1}-p\right\|^{2} \leq & \left(1-\alpha_{n}\right)^{2}\left\|x_{n}-p\right\|^{2}+2 \alpha_{n} \varepsilon_{n} \\
& +2 \alpha_{n} k_{n}\left\|x_{n+1}-p\right\|^{2}-2 \alpha_{n} \varphi\left(\left\|x_{n+1}-p\right\|\right) \\
& +h_{n} \alpha_{n} .
\end{aligned}
$$

Using (33) and $\left\|x_{n}-p\right\| \leq 2 \varphi^{-1}\left(a_{0}\right)=b_{0}$, we have

$$
\begin{aligned}
\left\|x_{n+1}-p\right\|^{2} \leq & \frac{\left(1-\alpha_{n}\right)^{2}}{1-2 \alpha_{n} k_{n}}\left\|x_{n}-p\right\|^{2}+\frac{\left(C_{n}+2 \varepsilon_{n}+h_{n}\right) \alpha_{n}}{1-2 \alpha_{n} k_{n}} \\
& -\frac{2 \alpha_{n}}{1-2 \alpha_{n} k_{n}} \varphi\left(\left\|x_{n+1}-p\right\|\right) \\
= & \left\|x_{n}-p\right\|^{2}+\frac{\left(-2+\alpha_{n}+2 k_{n}\right) \alpha_{n}}{1-2 \alpha_{n} k_{n}}\left\|x_{n}-p\right\|^{2} \\
& +\frac{\left(C_{n}+2 \varepsilon_{n}+h_{n}\right) \alpha_{n}}{1-2 \alpha_{n} k_{n}} \\
& -\frac{2 \alpha_{n}}{1-2 \alpha_{n} k_{n}} \varphi\left(\left\|x_{n+1}-p\right\|\right) \\
\leq & \left\|x_{n}-p\right\|^{2}+\frac{\left(-2+\alpha_{n}+2 k_{n}\right) \alpha_{n}}{1-2 \alpha_{n} k_{n}} b_{0}^{2} \\
& +\frac{\left(C_{n}+2 \varepsilon_{n}+h_{n}\right) \alpha_{n}}{1-2 \alpha_{n} k_{n}} \\
& -\frac{2 \alpha_{n}}{1-2 \alpha_{n} k_{n}} \varphi\left(\left\|x_{n+1}-p\right\|\right) .
\end{aligned}
$$

Since $1-2 \alpha_{n} k_{n} \rightarrow 1,(n \rightarrow \infty)$, there exists nonnegative integer $n_{0}>0$, such that when $n \geq n_{0},(1 / 2) \leq 1-2 \alpha_{n} k_{n} \leq 1$. Without loss of generality, for all $n \geq 0,(1 / 2) \leq 1-2 \alpha_{n} k_{n} \leq 1$. Let $\eta_{n}=\left(-2+\alpha_{n}+2 k_{n}\right) b_{0}^{2}+C_{n}+2 \varepsilon_{n}+h_{n} \rightarrow 0,(n \rightarrow \infty)$. From (34), for all $n \geq n_{2}$, we have

$$
\left\|x_{n+1}-p\right\|^{2} \leq\left\|x_{n}-p\right\|^{2}+2 \eta_{n} \alpha_{n}-2 \alpha_{n} \varphi\left(\left\|x_{n+1}-p\right\|\right) .
$$

Substituting (23) in (35), we have

$$
\left\|x_{n+1}-p\right\|^{2} \leq\left\|x_{n}-p\right\|^{2}+2 \eta_{n} \alpha_{n}-2 \alpha_{n} \varphi\left(b_{0}\right) .
$$

Since $\eta_{n} \rightarrow 0,(n \rightarrow \infty)$, there exists $n_{3} \geq n_{2}$, for all $n \geq n_{3}$, $\eta_{n} \leq\left(\varphi\left(b_{0}\right) / 2\right)$. Without loss of generality, for all $n \geq n_{2}, \eta_{n} \leq$ $\left(\varphi\left(b_{0}\right) / 2\right)$. So, from (36) and the condition (iii), we have

$$
\begin{gathered}
\left\|x_{n+1}-p\right\|^{2} \leq\left\|x_{n}-p\right\|^{2}-\alpha_{n} \varphi\left(b_{0}\right), \quad \forall n \geq n_{2}, \\
\alpha_{n} \varphi\left(b_{0}\right) \leq\left\|x_{n}-p\right\|^{2}-\left\|x_{n+1}-p\right\|^{2}, \\
+\infty=\varphi\left(b_{0}\right) \sum_{n=n_{2}}^{\infty} \alpha_{n} \leq\left\|x_{n_{2}}-p\right\|^{2} .
\end{gathered}
$$

This is a contradiction. Therefore for all $n \geq n_{2}$, we have $\| x_{n}-$ $p \| \leq 2 \varphi^{-1}\left(a_{0}\right)$.

Again, by (35) and the conditions (ii), (iii), we have

$$
\left\|x_{n+1}-p\right\|^{2} \leq\left\|x_{n}-p\right\|^{2}-2 \alpha_{n} \varphi\left(\left\|x_{n+1}-p\right\|\right)+2 \eta_{n} \alpha_{n} .
$$

Consider $\lim _{n \rightarrow \infty} \alpha_{n}=0, \lim _{n \rightarrow \infty}\left(2 \eta_{n} \alpha_{n} / 2 \alpha_{n}\right)=$ $\lim _{n \rightarrow \infty} \eta_{n}=0$, and $\sum_{n=0}^{\infty} \alpha_{n}=\infty$. So by Lemma 8 , we obtain $\lim _{n \rightarrow \infty}\left\|x_{n}-p\right\|=0$; that is, $x_{n} \rightarrow p$.

Proof (necessity). Assume that $x_{n} \rightarrow p \in F(T)$. Since $x_{n} \in$ $G$ and $T: G \rightarrow G$ is an asymptotically quasi- pseudocontractive-type mapping, we have

$$
\begin{array}{r}
\limsup _{n \rightarrow \infty} \inf _{j\left(x_{n+1}-p\right) \in J\left(x_{n+1}-p\right)}\left[\left\langle T^{n} x_{n+1}-p, j\left(x_{n+1}-p\right)\right\rangle\right. \\
\left.-k_{n}\left\|x_{n+1}-p\right\|^{2}\right] \leq 0 .
\end{array}
$$

For any given strictly increasing function $\varphi(s)$ with $\varphi(0)=0$, such as $\varphi(s)$, we have

$$
\lim _{n \rightarrow \infty} \varphi\left(\left\|x_{n+1}-p\right\|\right)=0 .
$$

From (39) and (40), we know that (12) holds.

Theorem 10. Let $G$ be a nonempty convex subset of a real Banach space $E$, and let $T: G \rightarrow G$ be an asymptotically nonexpansive in the intermediate sense and an asymptotically quasi-pseudo-contractive-type mapping with sequence $\left\{k_{n}\right\} \subset$ $[1, \infty), \lim _{n \rightarrow \infty} k_{n}=1$. Assume that $\left\{\alpha_{n}\right\}$ and $\left\{\gamma_{n}\right\}$ are two sequences in $[0,1]$ satisfying the following conditions:

(i) $\alpha_{n}+\gamma_{n} \leq 1$;

(ii) $\alpha_{n} \rightarrow 0,(n \rightarrow \infty)$;

(iii) $\gamma_{n}=o\left(\alpha_{n}\right), \sum_{n=0}^{\infty} \alpha_{n}=+\infty$.

Let $x_{0} \in G$ be a given point and let the sequence $\left\{x_{n}\right\}$ be the modified Mann iterative sequence with errors defined by (9). Then $\left\{x_{n}\right\}$ converges strongly to a fixed point $p \in F(T)$ if only if there exists a strictly increasing function $\varphi:[0, \infty) \rightarrow[0, \infty)$ with $\varphi(0)=0$ such that the condition (12) of Theorem (12) holds.

Remark 11. Our research and results in this paper have the following advantages. (a) The iterative scheme is the modified mixed Ishikawa and Mann iterative scheme with error. (b) The research object is the very generalized asymptotically quasi-pseudo-contractive-type mappings. (c) The proof methods are very different from previous ones and we do not need the condition of "boundedness of $T$ " (e.g., [4]). So, the results here generalize and improve the recent results announced by many other authors to a certain extent, such as $[1,4,5,12,13]$.

\section{Conflict of Interests}

The authors declare that there is no conflict of interests regarding the publication of this paper. 


\section{Acknowledgments}

The authors would like to thank the editors and referees for many useful comments and suggestions for the improvement of the paper. This work was supported by the National Natural Science Foundation of China (Grant no. 11271330) and the Natural Science Foundation of Zhejiang Province (Grant no. Y6100696).

\section{References}

[1] S. S. Chang, "Some results for asymptotically pseudocontractive mappings and asymptotically nonexpansive mappings," Proceedings of the American Mathematical Society, vol. 129, no. 3, pp. 845-853, 2001.

[2] K. Goebel and W. A. Kirk, "A fixed point theorem for asymptotically nonexpansive mappings," Proceedings of the American Mathematical Society, vol. 35, pp. 171-174, 1972.

[3] J. Schu, "Iterative construction of fixed points of asymptotically nonexpansive mappings," Journal of Mathematical Analysis and Applications, vol. 158, no. 2, pp. 407-413, 1991.

[4] L. Zeng, "Modified Ishikawa iteration process with errors in Banach," Acta Mathematica Sinica, vol. 47, no. 2, pp. 219-228, 2004.

[5] L. Zeng, "On the strong convergence of iterative method for non-Lipschitzian asymptotically pseudocontractive mappings," Acta Mathematicae Applicatae Sinica, vol. 27, no. 3, pp. 230-239, 2004.

[6] Y. Yao, R. Chen, G. Marino, and Y.-C. Liou, "Applications of fixed point and approximate algorithms," Journal of Applied Mathematics, vol. 2012, Article ID 175459, 2 pages, 2012.

[7] L. Wei, Y.-F. Su, and H.-Y. Zhou, "New iterative schemes for strongly relatively nonexpansive mappings and maximal monotone operators," Applied Mathematics, vol. 25, no. 2, pp. 199-208, 2010.

[8] D. Zhang, X. Qin, and F. Gu, "Approximation of common fixed points of nonexpansive semigroups in Hilbert spaces," Journal of Applied Mathematics, vol. 2012, Article ID 417234, 13 pages, 2012.

[9] L.-C. Ceng and C.-F. Wen, "Composite iterative algorithms for variational inequality and fixed point problems in real smooth and uniformly convex Banach spaces," Journal of Applied Mathematics, vol. 2013, Article ID 761864, 21 pages, 2013.

[10] L.-C. Ceng, H.-K. Xu, and C.-F. Wen, "Relaxed viscosity approximation methods with regularization for constrained minimization problems," Journal of Applied Mathematics, vol. 2013, Article ID 531859, 19 pages, 2013.

[11] L.-C. Ceng and C.-F. Wen, "Hybrid method with perturbation for Lipschitzian pseudo-contractions," Journal of Applied Mathematics, vol. 2012, Article ID 250538, 20 pages, 2012.

[12] R. E. Bruck, T. Kuczumow, and S. Reich, "Convergence of Iterates of asymptotically nonexpansive mappings in Banach spaces with the uniform Opial property," Colloquium Mathematicum, vol. 65 , no. 2, pp. 169-179, 1993.

[13] L. Zeng, "On the Iterative construction of fixed points of asymptotically pseudo-contractive type mappings," Journal of Systems Science and Mathematical Sciences, vol. 24, pp. 261-270, 2004.

[14] Y. Wang, "Strong convergence theorems for asymptotically weak $G$-pseudo- $\psi$-contractive non-self-mappings with the generalized projection in Banach spaces," Abstract and Applied Analysis, vol. 2012, Article ID 651304, 11 pages, 2012.
[15] C. Moore and B. V. C. Nnoli, "Iterative solution of nonlinear equations involving set-valued uniformly accretive operators," Computers and Mathematics with Applications, vol. 42, no. 1-2, pp. 131-140, 2001. 


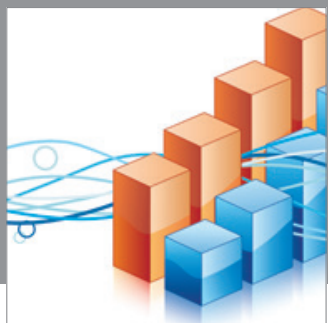

Advances in

Operations Research

mansans

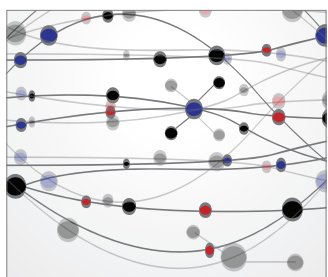

The Scientific World Journal
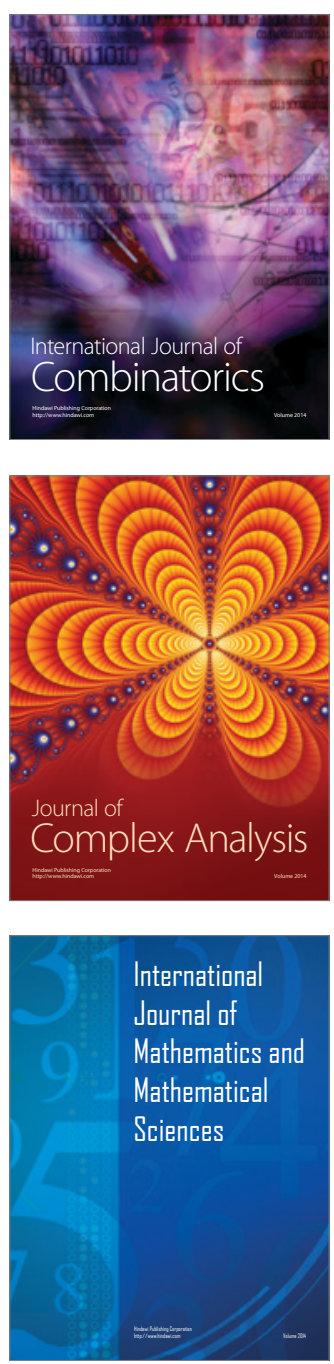
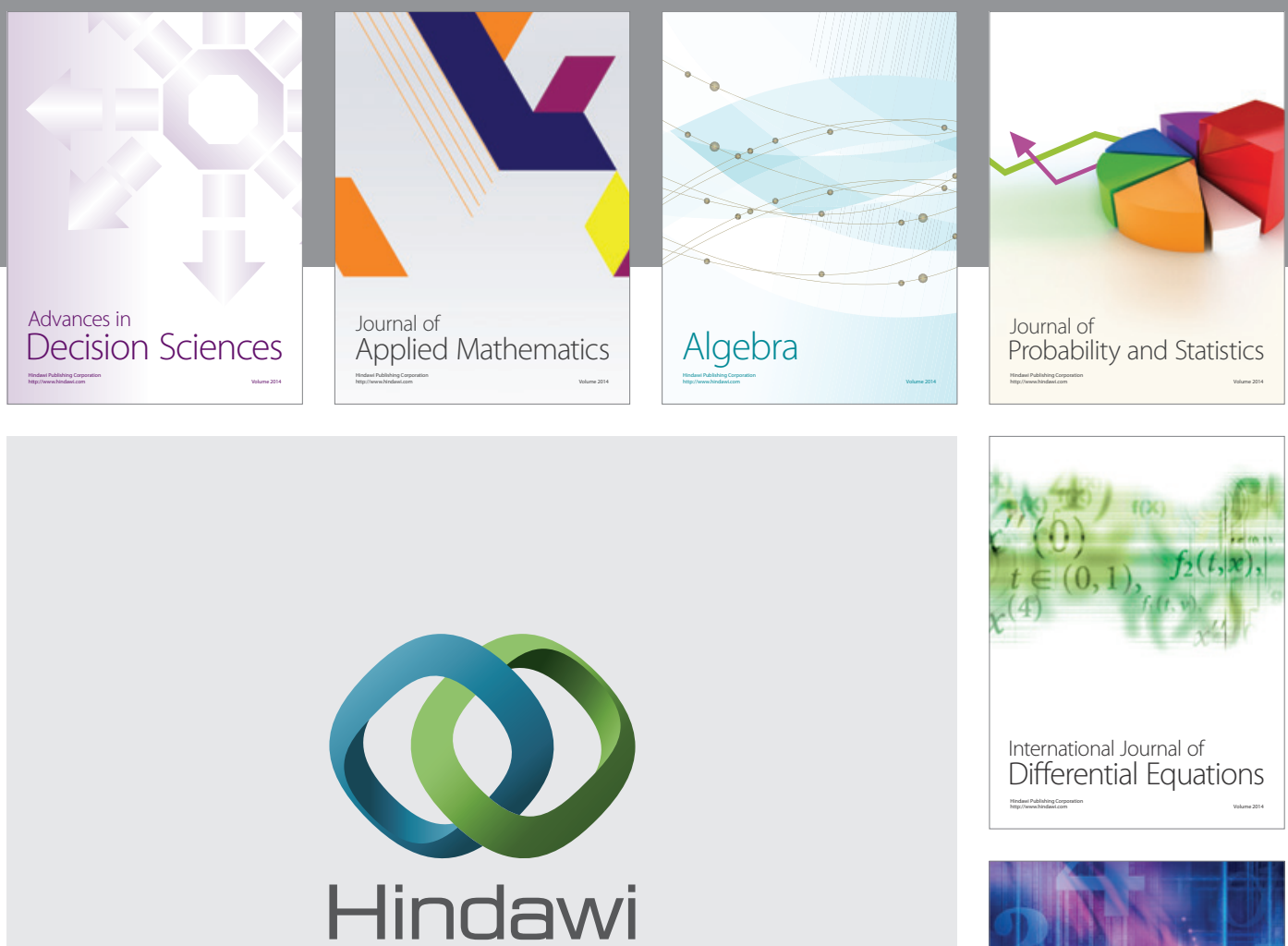

Submit your manuscripts at http://www.hindawi.com
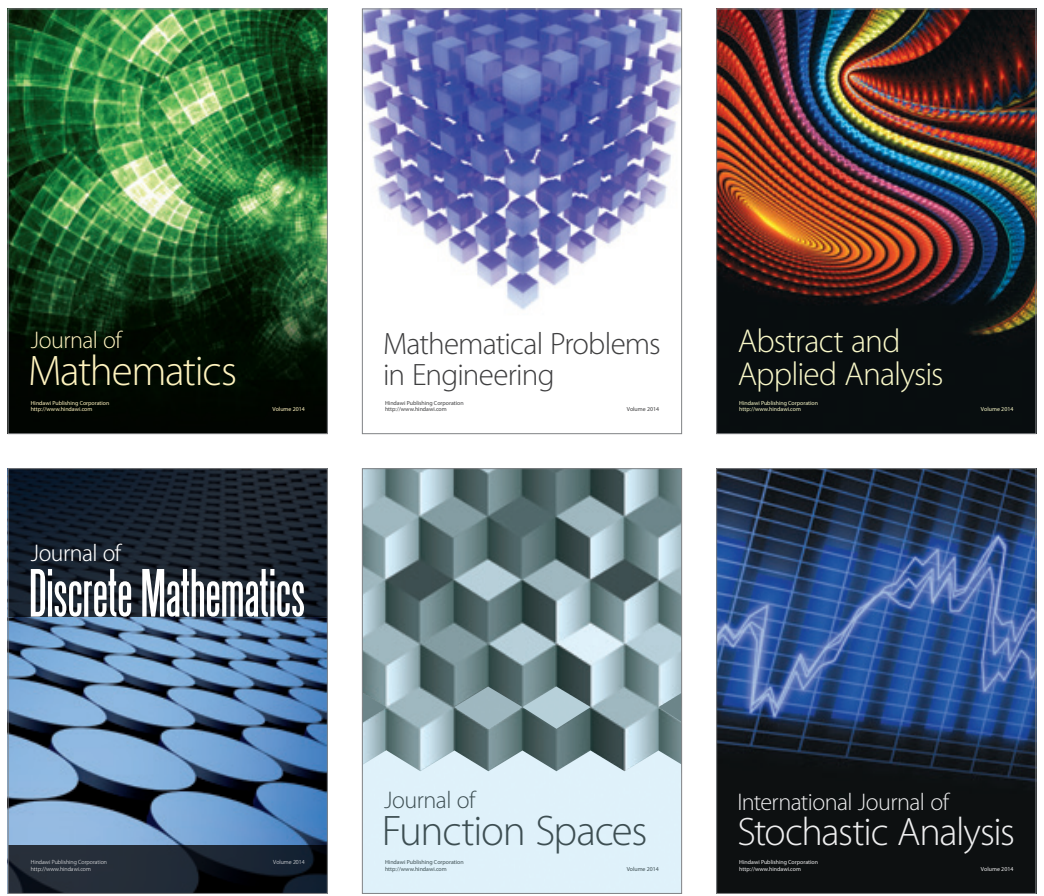

Journal of

Function Spaces

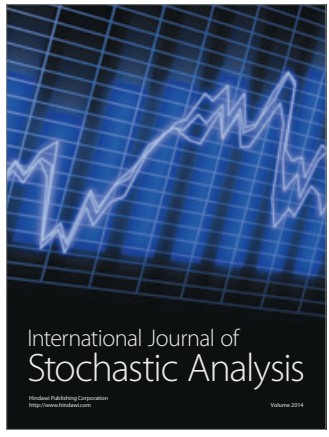

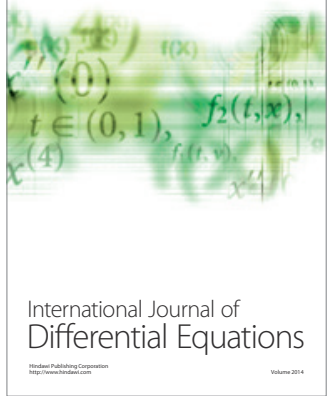
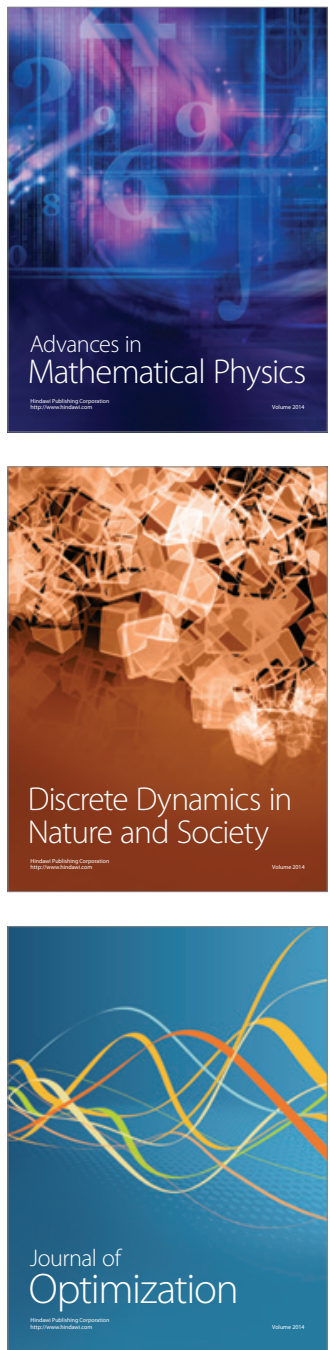